\title{
Correlation for Surfaces of General Type
}

\author{
Brendan Hassett*
}

October 29, 2018

This paper is still in rough form, and I will appreciate any comments on the content or the exposition.

\section{Introduction}

The purpose of this paper is to prove the following theorem:

Theorem 1 (Correlation Theorem for Surfaces) Let $f: X \longrightarrow B$ be a proper morphism of integral varieties, whose general fiber is an integral surface of general type. Then for $n$ sufficiently large, $X_{B}^{n}$ admits a dominant rational map $h$ to a variety $W$ of general type such that the restriction of $h$ to a general fiber of $f^{n}$ is generically finite.

This theorem has a number of geometric and number theoretic consequences that will be discussed in the final section of this paper. In particular, assuming Lang's conjecture on rational points of varieties of general type, we can prove a uniform bound on the number of rational points on a surface of general type not contained in rational or elliptic curves.

This theorem is a special case of the following conjecture posed by Caporaso, Harris, and Mazur [CHM]:

Conjecture 1 (Correlation Conjecture) Let $f: X \longrightarrow B$ be a proper morphism of integral varieties, whose general fiber is an integral variety of general type.

*This work was partially supported by a National Science Foundation Graduate Fellowship 
Then for $n$ sufficiently large, $X_{B}^{n}$ admits a dominant rational map $h$ to a variety $W$ of general type such that the restriction of $h$ to a general fiber of $f^{n}$ is generically finite.

They prove this conjecture in the case where the general fiber is a curve of genus $g \geq 2$. This implies that if Lang's conjectures on the distribution of rational points on varieties of general type are true, then there is a uniform bound on the number of rational points on a curve of genus $g$ defined over a number field $K$. The paper [CHM] contains most of the ingredients necessary for a proof of the general conjecture. However, at one point the argument relies heavily on the fact that the fibers of the map are curves: it invokes the existence of a 'nice' class of singular curves, the stable curves. For the purposes of this discussion, 'nice' means two things:

1. Given any proper morphism $f: X \longrightarrow B$ whose generic fiber is a smooth curve of genus $g \geq 2$, there exists a generically finite base change $B^{\prime} \longrightarrow B$ so that the dominating component of $X \times_{B} B^{\prime}$ is birational to a family of stable curves over $B^{\prime}$.

2. Let $f: X \rightarrow B$ be a family of stable curves, smooth over the generic point. Then the fiber products $X_{B}^{n}$ are canonical.

For the purpose of generalizing to higher dimensions, we make the following definitions:

Let $\mathcal{C}$ be a class of singular varieties.

$\mathcal{C}$ is inclusive if for any proper morphism $f: X \rightarrow B$ whose generic fiber is a variety of general type, there is a generically finite base change $B^{\prime} \rightarrow B$ such that $X \times_{B} B^{\prime}$ is birational to a family $X^{\prime} \rightarrow B^{\prime}$ with fibers in $\mathcal{C}$.

$\mathcal{C}$ is negligible if for any family of varieties of general type $f: X \rightarrow B$ with singular fibers belonging to $\mathcal{C}$, the fiber products $X_{B}^{n}$ have canonical singularities.

In a nutshell, the main obstruction to extending the results of [CHM] is to find a class of higher dimensional singular varieties which is both negligible and inclusive. In this paper, we identify a class of surface singularities which is both inclusive and negligible, and prove that the class has these properties. 
This is the class of 'stable surfaces', surfaces at the boundary of a compactification of the moduli space of surfaces of general type.

In the second section of this paper, we describe these stable surfaces, and try to motivate their definition. In the third section, we prove that these stable surfaces actually form an inclusive class of singularities. In the fourth section, we prove that stable surfaces are negligible, i.e. that their fiber products have canonical singularities. In the fifth section, we sketch the proof of the Correlation Theorem for surfaces of general type outlined in CHM. Finally, we state some consequences of the Correlation Theorem, assuming various forms of the Lang conjectures.

I would like to thank Joe Harris for suggesting this problem, and Dan Abramovich for his countless comments and corrections. János Kollár also provided useful suggestions for the results in section four.

Thorughout this paper, we work over a field of characteristic zero.

\section{Stable Surfaces}

In this section, we describe the class of stable surfaces and their singularities. Most of these results are taken from [K-SB] and [K].

Stable surfaces are defined so that one has a stable reduction theorem for surfaces, analogous to stable reduction for curves. For motivation, we first review the curve case. Let $f: X \rightarrow \Delta$ be a flat family of curves of genus $g \geq 2$ over a disc. Assume that the fibers of the family are smooth, except for

the fiber $f^{-1}(0)$ which may be singular. By Mumford semistable reduction $([\mathrm{KKMS}])$, there is a finite base change

$$
\tilde{\Delta} \longrightarrow \Delta
$$

ramified over 0 , and a resolution of singularities of the base-changed family

$$
d: Y \longrightarrow \tilde{X}
$$

such that the fibers of the composed map

$$
F=d \circ \tilde{f}: Y \longrightarrow \tilde{\Delta}
$$


are reduced normal crossings divisors. This semistable reduction is not unique, as we can always blow up $Y$ to get a 'different' semistable reduction. These semistable reductions are all birational, and we can take a 'canonical model' $\mathcal{Y}$ of the surface $Y$ by using the relative pluricanonical differentials to map $Y$ birationally into projective space. $\mathcal{Y}$ is the image of this map, and $\mathcal{Y} \rightarrow \tilde{\Delta}$ is called the stable reduction of our original family. Moreover, the birational map $Y \rightarrow \mathcal{Y}$ can be described quite explicity. It is the morphism that blows down all the -1 and -2 curves on $Y$. On the fibers, this corresponds to blowing down smooth rational components meeting the rest of the fiber in one or two points. From this, we see that the fibers of $\mathcal{Y} \rightarrow \tilde{\Delta}$ are just stable curves.

For higher dimensional varieties, we can try to mimic the same procedure. We can still apply semistable reduction to obtain the family $Y \longrightarrow \tilde{\Delta}$, but this reduction is not unique. The problem is that it is not generally known how to obtain a canonical model $\mathcal{Y}$ for the birational equivalence class of $Y$. This canonical model $\mathcal{Y}$ would be our stable reduction, if it were well defined. In the case of families of surfaces where $Y$ is a threefold, we can use the minimal model program to construct the canonical model of a semistable family of surfaces (cf $\mathbb{K a}$ ). The total space of our stable family will then have canonical singularities, and the singularities of the fibers of the family can then be described.

Now we introduce the formal definitions. By definition, a variety $S$ is said to be $\mathbb{Q}$-Gorenstein if $\omega_{S}^{[k]}$ is locally free for some $k$. $\omega_{S}^{[k]}$ denotes the reflexive hull (i.e. the double dual) of the $k$ th power of the dualizing sheaf. For a $\mathbb{Q}$-Gorenstein singularity, the smallest such $k$ is called the index of the singularity. A surface is semi-smooth if it has only the following singularities:

1. 2-fold normal crossings with equation $x^{2}=y^{2}$

2. pinch points with equation $x^{2}=z y^{2}$

A good semi-resolution resolution of $S$ is a proper map $g: T \longrightarrow S$ satisfying the following properties

1. $T$ is semi-smooth

2. $g$ is an isomorphism in the complement of a codimension two subscheme of $T$ 
3. No component of the double curve of $T$ is exceptional for $g$.

4. The components of the double curve of $T$ and the exceptional locus of $S$ are smooth, and meet transversally.

A surface $S$ is said to have semi-log-canonical singularities if

1. $S$ is Cohen-Macaulay and $\mathbb{Q}$-Gorenstein with index $k$

2. $S$ is semi-smooth in codimension one

3. The discrepancies of a good semi-smooth resolution of $S$ are all greater than or equal to -1 (i.e. $\omega_{T}^{k}=g^{*} \omega_{S}^{[k]}\left(k a_{1} E_{1}+\ldots+k a_{n} E_{N}\right)$ where $\left.a_{i} \geq-1\right)$

In [K-SB] a complete classification of semi-log-canonical singularities is given. The relevance of these definitions comes from a result proved in the same paper

Theorem 2 Let $f: X \rightarrow \Delta$ be a family of surfaces over the disc. Then the following are equivalent:

1. The general fiber has rational double points, and the central fiber has semi-log-canonical singularities.

2. For any base change $\tilde{\Delta} \rightarrow \Delta$, the base-changed family

$$
\tilde{f}: \tilde{X} \longrightarrow \tilde{\Delta}
$$

has canonical singularities.

In fact, if $X \rightarrow \Delta$ has a semistable resolution of singularities, then $X$ is canonical iff the general fiber has rational double points and the central fiber has semi-log-canonical singularities.

In particular, this means that the 'bad' fibers in a stable reduction of surfaces have only semi-log-canonical singularities. For the sake of this discussion, surfaces with only rational double point singularities are 'good' fibers. This is reasonable, because we would like the canonical model of a smooth surface of general type to be 'good'. This motivates the definition: 
A surface $S$ is stable if $S$ has semi-log-canonical singularities, and for some sufficiently large $k \omega_{S}^{[k]}$ is locally free and ample.

Note that a smooth surface of general type is not stable if it contains -1 or -2 curves, but its canonical model will be stable.

A family of stable surfaces is defined to be a proper flat morphism $\mathcal{S} \rightarrow \mathcal{B}$ whose fibers are stable surfaces, with the property that taking reflexive powers of the relative dualizing sheaf commutes with restricting to a fiber:

$$
\omega_{\mathcal{S} / \mathcal{B}}^{[k]} \mid \mathcal{S}_{b}=\omega_{\mathcal{S}_{b}}^{[k]}
$$

In particular, the reflexive powers of the relative dualizing sheaf are flat. This additional condition is necessary to guarantee that the moduli space in the next section is separated. Note that we can define

$$
K_{S}^{2}=\frac{1}{k^{2}} \#\left(\omega_{S}^{[k]}, \omega_{S}^{[k]}\right)
$$

for any stable surface $S$, and that this number is constant in families. We also have the invariant $\chi_{S}=\chi\left(\mathcal{O}_{\mathcal{S}}\right)$, which is also constant in families. Finally, stable surfaces are analogous to stable curves in one more important sense:

Theorem 3 A stable surface has a finite automorphism group.

The essence of the proof is easy to grasp. Let $S$ be stable, and let $\tilde{S}$ be its normalization. Let $\Delta$ be the double curve on $\tilde{S}$. The pair $(\tilde{S}, \Delta)$, is $\log$ canonical (see $[\bar{K}-\mathrm{SB}]$ ). Therefore, each component of $(\tilde{S}, \Delta)$ is of log-general type, and has a finite automorphism group by [1].

\section{Stable Surface Singularities are Inclusive}

To show that the class of stable surfaces are inclusive, we need to invoke the existence of a proper coarse moduli space $\overline{\mathcal{M}}_{\chi, K^{2}}$ for the stable surfaces with invariants $\chi$ and $K^{2}$. We also need a finite covering $\phi: \Omega \rightarrow \overline{\mathcal{M}}_{\chi, K^{2}}$ of the moduli space that admits a tautological family $\mathcal{S} \rightarrow \Omega$ :

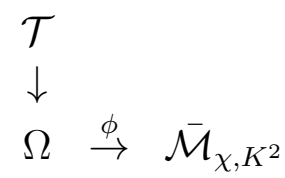


We have the following theorem:

Theorem 4 For smoothable stable surfaces, there exists a coarse moduli space $\overline{\mathcal{M}}_{\chi, K^{2}}$ with these properties.

By definition, a stable surface is smoothable if it is contained in a family of stable surfaces with $\mathbb{Q}$-Gorenstein total space, such that the general member has only rational double points. The proof of this theorem is scattered throughout the literature. The proof that the moduli space exists as a separated algebraic space is contained in $[\mathrm{K}-\mathrm{SB}$ §5. This relies on the properties of semi-log-canonical singularities and the finite automorphism theorem. The proof that the moduli space has a functorial semipositive polarization is contained in [K] §5. This paper also has a general argument for the existence of a finite covering of the moduli space possessing a tautological family (see also CHM §5.1). The proof that the moduli space is of finite type for a given pair of invariants (and thus proper and projective by [《]) is contained in $\mathrm{A}$.

Using this moduli space, we can prove that the class of stable surface singularities is inclusive.

Proposition 1 Let $f: X \rightarrow B$ be a proper morphism of integral varieties. Assume that the general fiber of this map is a smooth surface of general type. Then there exists a generically finite base change $B^{\prime} \rightarrow B$ such that the dominating component of the fiber product $X \times_{B} B^{\prime}$ is birational to a family of stable surfaces over $B^{\prime}$.

The proof of this follows the proof of the analogous result in CHM $§ 5.2 \mathrm{t}$ quite closely. Since $\overline{\mathcal{M}}$ is a coarse moduli space, there is an induced rational map $B \rightarrow \overline{\mathcal{M}}$. Let $B_{1}$ be the closed graph of this map, $\Sigma_{1}$ its image in $\overline{\mathcal{M}}$, and $X_{1} \rightarrow B_{1}$ the dominating component of $X \times_{B} B_{1}$. Since there is no tautological family on $\overline{\mathcal{M}}$, we do not have a family of stable surfaces defined over $\Sigma_{1}$. However, we do have such a family over $\Sigma_{2}=\phi^{-1}\left(\Sigma_{1}\right) \subset \Omega$, which we denote $\mathcal{T}_{\epsilon}$. So we let $B_{2}=B_{1} \times_{\Sigma_{1}} \Sigma_{2}, \mu: B_{2} \rightarrow \Sigma_{2}$ the projection, and $X_{2} \rightarrow B_{2}$ the main component of $X_{1} \times_{B_{1}} B_{2}$. The family $\mathcal{T}_{\in}$ of stable surfaces pulls back to a family $Y_{2}=\mathcal{T}_{\in} \times_{\Sigma_{\in}} \mathcal{B}_{\in} \rightarrow \mathcal{B}_{\in}$. For general $b \in B_{2},\left(X_{2}\right)_{b}$ is birational to $\left(Y_{2}\right)_{b}$ by construction.

This is almost enough to prove that $X_{2}$ is birational to the family of stable surfaces $Y_{2}$. We just need one further finite base change $B_{3} \rightarrow B_{2}$ to 
'straighten out' $X_{2}$. We have to get rid of isotrivial subfamilies that cannot be represented by pull backs of the tautological family on the moduli space. We describe the fiber of this base change over the generic point $b \in B_{2}$. Set theoretically, it will correspond to equivalence classes of birational morphisms

$$
\left\{\psi \mid \psi:\left(X_{2}\right)_{b} \rightarrow\left(\mathcal{T}_{\in}\right)_{\mu(\mathrm{L})}\right\}
$$

Two maps are equivalent if they induce the identity on the canonical model $\left(\mathcal{T}_{\epsilon}\right)_{\mu(\downarrow)}$. The algebraic structure is just the natural algebraic structure on the finite automorphism group of the stable surface. If our covering variety happens to be disconnected, choose a component $B^{\prime \prime}$ dominating $B_{2}$. Finally, we take $B_{3} \rightarrow B$ to be the Galois normalization of $B^{\prime \prime} \rightarrow B$, and let $G$ be Galois group $\operatorname{Gal}\left(k\left(B_{3}\right) / k(B)\right)$. We let $X_{3}$ denote the principal component of $X_{2} \times_{B_{2}} B_{3}$. We can represent a generic point of $X_{3}$ as a triple

$$
(p, b, \psi)
$$

where $b \in B_{2}, p \in\left(X_{2}\right)_{b}$, and $\psi:\left(X_{2}\right)_{b} \rightarrow\left(\mathcal{T}_{\in}\right)_{\mu(\llcorner)}$. The birational map from $X_{3}$ to $Y_{3}=\mathcal{T}_{\in} \times_{\Sigma_{\epsilon}} \mathcal{B}_{\ni}$ is just the evaluation map

$$
(p, b, \psi) \longrightarrow(\psi(p), b, \psi)
$$

Setting $B^{\prime}=B_{3}$, we obtain the proposition that stable surface singularities are inclusive.

For the proof of the Correlation Theorem, we will need to elaborate a bit on this situation. Let $\Sigma_{3} \rightarrow \Sigma_{1}$ denote the Galois normalization of $\Sigma_{1}$ in the function field $k\left(B_{3}\right)$. We have:

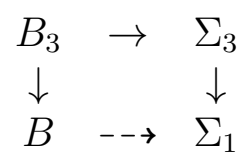

The bottom arrow is only birational. Note that the map $B_{3} \rightarrow \Sigma_{2}$ factors naturally through $\Sigma_{3}$; this is just the Stein factorization. We claim that $G$ acts naturally on this diagram. $G$ consists of automorphisms of $k\left(B_{3}\right)$ fixing the subfield $k\left(B_{1}\right)$. Since $k\left(\Sigma_{1}\right) \subset k\left(B_{1}\right)$ these automorphisms fix $k\left(\Sigma_{1}\right)$ as well, and they restrict to automorphisms of the elements of $k\left(B_{3}\right)$ algebraic over $k\left(\Sigma_{1}\right)$, i.e. $k\left(\Sigma_{3}\right)$.

Now let $\mathcal{T}_{3}$ denote $\mathcal{T}_{2} \times_{\Sigma_{2}} \Sigma_{3}$. We shall show that $G$ also acts birationally 
and equivariantly on $\mathcal{T}_{3} \rightarrow \Sigma_{3}$. Let $s \in \Sigma_{3}$ be a general point, and $\left(\mathcal{T}_{3}\right)_{s}$ the corresponding fiber. For $g \in G$, we need to describe the map

$$
\left(\mathcal{T}_{3}\right)_{s} \rightarrow\left(\mathcal{T}_{3}\right)_{g(s)}
$$

By construction $\left(\mathcal{T}_{3}\right)_{s}$ can be birationally identified with some corresponding fiber of $X_{3} \rightarrow B_{3}$. The action of $g$ maps this surface to another fiber of $X_{3} \rightarrow B_{3}$, which in turn can be birationally identifed with $\left(\mathcal{T}_{3}\right)_{g(s)}$. This gives a commutative diagram of varieties with (birational) G-actions

$$
\begin{array}{cccc}
X_{3} & \rightarrow & \mathcal{T}_{3} \\
\downarrow & & \downarrow \\
B_{3} & \rightarrow & \Sigma_{3}
\end{array}
$$

From the arguments in the previous paragraph we see that $X_{3}$ is birational to $Y_{3}=\mathcal{T}_{3} \times{ }_{\Sigma_{3}} B_{3}$, and that this birational map respects the Galois action of $G$. Taking quotients under this action gives a dominant rational map

$$
X \approx\left(\mathcal{T}_{3} \times_{\Sigma_{3}} B_{3}\right) / G \longrightarrow \mathcal{T}_{3} / G
$$

This refined construction is crucial to the proof of the correlation theorem, so we summarize it below:

Corollary 1 Let $f: X \rightarrow B$ be a proper morphism of integral varieties. Assume that the general fiber of $f$ is a surface of general type. Then there exists a generically finite Galois base extension

$$
B^{\prime} \rightarrow B
$$

with Galois group $G$, and a finite cover of the image of $B$ in the moduli space

$$
\Sigma^{\prime} \rightarrow \Sigma
$$

with the following properties:

1. There is a tautological family of surfaces

$$
\mathcal{T}^{\prime} \rightarrow \Sigma^{\prime}
$$

over $\Sigma^{\prime}$. 
2. $G$ acts on $\Sigma^{\prime}$, and this action lifts to a $G$ equivariant rational dominant map

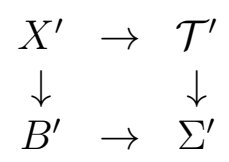

3. The pull back of $\mathcal{T}^{\prime}$ to $B^{\prime}$ is birational to $X^{\prime}$, and the quotient of this variety under the $G$-action is birational to $X$.

\section{Stable Surface Singularities are Negligible}

In this section, we will restrict our attention to families of stable surfaces $f: X \rightarrow B$ over a smooth base $B$, and their fiber products $f^{n}: X_{B}^{n}=$ $X \times_{B} \ldots \times_{B} X \rightarrow B$. For such families, having canonical singularities (rational double points) is an open condition. That is, the locus $S \subset B$ corresponding to singularities worse than rational double points is Zariski closed. Here we will assume that it is a proper subvariety of $B$. We will prove the following:

Proposition 2 Let $f: X \rightarrow B$ be a family of stable surfaces over a smooth proper base B. Assume the generic fiber has only canonical singularities. Then the fiber products of this family

$$
f^{n}: X_{B}^{n} \longrightarrow B
$$

have canonical singularities.

We prove this in three steps. First, we prove a general lemma on the singularities of fiber products. Then we establish the result in the case where $B=\Delta$ a complex disc. The third step is to reduce the general case to this special case. For this reduction, we will utilize results of Stevens [St] on families of varieties with canonical singularities.

Our first lemma gives some rough information on the singularities of fiber products:

Lemma 1 Let $f: X \rightarrow B$ a family of stable surfaces, such that the general fiber is normal. Then the $n^{\text {th }}$ fiber product

$$
f^{n}: X_{B}^{n} \longrightarrow B
$$

is a normal $\mathbb{Q}$-Gorenstein variety. 
$X_{B}^{n}$ is irreducible, because the family $X \rightarrow B$ is flat with general fiber irreducible. We show that $X_{B}^{n}$ is Cohen-Macaulay. $X_{B}$ itself is Cohen-Macaulay, as it is a flat family of Cohen-Macaulay varieties over a smooth base. In particular, the dualizing complex of the morphism $X \rightarrow B$ has only a single term, the relative dualizing sheaf $\omega_{X / B}$. This sheaf is flat over $B$, so the dualizing complex of $X_{B}^{n} \rightarrow B$ is just the tensor product of the dualizing complexes of each of the factors. In particular, this complex has only one term

$$
\omega_{X_{B}^{n} / B}=\pi_{1}^{*} \omega_{X / B} \otimes \ldots \otimes \pi_{n}^{*} \omega_{X / B}
$$

and so $X_{B}^{n}$ is Cohen-Macaulay. Note that this implies that $X_{B}^{n}$ satisfies Serre's condition $S_{r}$ for every $r>0$.

$X_{B}^{n}$ is reduced, because it is smooth at the generic point and satisfies the $S_{1}$ condition. We now prove that $X_{B}^{n}$ is normal. Because $X_{B}^{n}$ satisfies the $S_{2}$ condition, we just need to show that it is smooth in codimension one. Let

$$
\pi_{j}: X_{B}^{n} \longrightarrow X
$$

be the $j^{\text {th }}$ projection map. The singularities of $X_{B}^{n}$ are contained in the set of points where $f^{n}$ fails to be a smooth morphism. But if $f^{n}$ fails to be smooth at $p$, then $f$ fails to be smooth at $\pi_{j}(p)$ for some $j$. Since $f$ is smooth on a set with codimension two complement, so is $f^{n}$. Thus the singularities of $X_{B}^{n}$ are in codimension two.

Now we prove the $\mathbb{Q}$ Gorenstein assertion. First we check that $X$ itself is $\mathbb{Q}$ Gorenstein, i.e. $\omega_{X}^{[N]}$ is locally free for some $N$. Since $X$ is a family of stable surfaces, there exists an integer $N$ such that for each $b \in B \omega_{X / B}^{[N]} \mid X_{b}$ is locally free. Since $\omega_{X / B}^{[N]}$ is free on every fiber of $X \rightarrow B, \omega_{X / B}^{[N]}$ is locally free. Since $B$ is smooth, $\omega_{B}$ is locally free, and

$$
\omega_{X}^{[N]}=\omega_{X / B}^{[N]} \otimes f^{*} \omega_{B}^{N}
$$

This formula is not hard to prove. It is certainly true on the open set $U$ where $X \rightarrow B$ is smooth. The complement of $U$ has codimension two by hypothesis. Since $X$ is normal, and both sheaves are reflexive, the formula extends to all of $X$. For the basic properties of reflexive sheaves used here, see $[\mathbb{H}]$.

Now we prove $X_{B}^{n}$ is $\mathbb{Q}$ Gorenstein. As in the previous paragraph, we have the formula

$$
\omega_{X_{B}^{n}}^{[N]}=\omega_{X_{B}^{n} / B}^{[N]} \otimes f^{n *} \omega_{B}^{\otimes N}
$$


so it suffices to prove that $\omega_{X_{B}^{n} / B}^{[N]}$ is locally free. Using the general formula:

$$
\omega_{X_{B}^{n} / B}=\pi_{1}^{*} \omega_{X / B} \otimes \ldots \otimes \pi_{n}^{*} \omega_{X / B} \quad(*)
$$

we will prove

$$
\omega_{X B}^{[N]}=\pi_{1}^{*} \omega_{X / B}^{[N]} \otimes \ldots \otimes \pi_{n}^{*} \omega_{X / B}^{[N]} \quad(* *)
$$

and so $\omega_{X_{B}^{n} / B}^{[N]}$ is locally free. The left hand side of $(* *)$ is reflexive by construction, and the right hand side is locally free because it is the tensor product of locally free sheaves. So we just need to prove the equivalence of the two sides of $(* *)$ on an open set with codimension two complement. Again, we choose the open set where $f^{n}$ is smooth as a morphism. On this set, the formula follows immediately from $(*)$, as the dualizing sheaves are already locally free. This completes the proof of the lemma.

Now we prove our proposition in the case where the base $B$ is one dimensional. In this special case it takes the following form:

Proposition 3 Let $f: X \rightarrow \Delta$ be a family of stable surfaces over the disc. Assume that the general fiber has only rational double points. Then the fiber products of this family over $\Delta$ have canonical singularities.

We apply semistable reduction to the family $X \rightarrow \Delta$. Let

$$
\tilde{\Delta} \longrightarrow \Delta
$$

be the ramified base change, and

$$
d: Y \longrightarrow \tilde{X}
$$

a resolution of singularities such that all the fibers of the induced map

$$
F=d \circ \tilde{f}: Y \longrightarrow \tilde{\Delta}
$$

are reduced normal crossings divisors. We have the diagram:

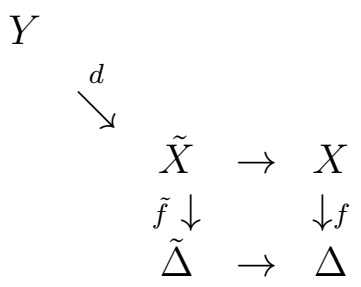


Using theorem 3 , we find that $\tilde{X}$ still has canonical singularities.

The next step is to take the nth fiber products of all the varieties in this diagram. We take the fiber products over the bases $\Delta$ and $\tilde{\Delta}$, and we use $f^{n}, \tilde{f}^{n}$, and $d^{n}$ to denote the maps on the fiber products induced by $f, \tilde{f}$, and $d$ respectively. We have the following diagram:

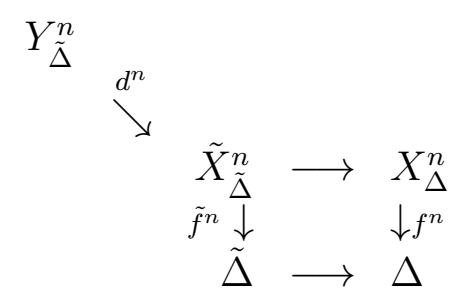

The general lemma implies that $X_{\Delta}^{n}$ and $\tilde{X}_{\tilde{\Delta}}^{n}$ are both $\mathbb{Q}$-Gorenstein and normal. As for $Y_{\tilde{\Delta}}^{n}$, recall that we constructed $Y$ so that its fibers over $\tilde{\Delta}$ have only reduced normal crossings. Using an argument of Viehweg [V] §3.6, we see that the singularities of $Y_{\tilde{\Delta}}^{n}$ are canonical. (Using local analytic coordinates, we can see that the singularities are toroidal, and so are rational. The equations also show that the singularities are local complete intersections, hence Gorenstein. But rational Gorenstein singularities are canonical).

First, note that for any $M$ there is an inclusion map:

$$
d_{*} \omega_{Y}^{M} \hookrightarrow \omega_{\tilde{X}}^{[M]}
$$

This is because the resolution $d: Y \rightarrow \tilde{X}$ is an isomorphism on an open set with codimension two in $\tilde{X}$, so pluricanonical forms on $Y$ yield sections of $\omega_{\tilde{X}}^{[M]}$. Moreover, since $\tilde{X}$ has canonical singularities we have that this is an isomorphism for some $M$, i.e.

$$
d_{*} \omega_{Y}^{M}=\omega_{\tilde{X}}^{[M]}
$$

This expresses the fact that regular pluricanonical differentials on the smooth locus of $\tilde{X}$ lift to smooth differentials on the desingularization $Y$. We will show:

$$
\omega_{\tilde{X}_{\tilde{\Delta}}^{n}}^{[M]}=d_{*}^{n} \omega_{Y_{\tilde{\Delta}}^{n}}^{M}
$$

This combined with the fact that $\tilde{X}_{\tilde{\Delta}}^{n}$ is $\mathbb{Q}$-Gorenstein and $Y_{\tilde{\Delta}}^{n}$ is canonical implies that $\tilde{X}_{\tilde{\Delta}}^{n}$ is canonical as well. 
We prove that (1) holds. Again, we have projection maps, which fit into a commutative diagram

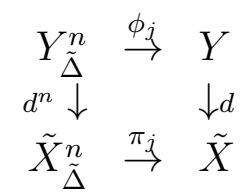

An important element in the proof (1) is the equation:

$$
d_{*}^{n} \phi_{j}^{*} \omega_{Y / \tilde{\Delta}}=\pi_{j}^{*} d_{*} \omega_{Y / \tilde{\Delta}}
$$

For simplicity, we prove this for $j=1$. We begin factoring $\phi_{1}=q \circ p$ and $d^{n}=r \circ p$ :

$$
\begin{array}{cccc}
Y \times \ldots \times Y & & \\
p \downarrow & \stackrel{\phi_{1}}{ } & \\
Y \times \tilde{X} \times \ldots \times \tilde{X} & \stackrel{\searrow}{\rightarrow} & Y \\
r \downarrow & & \downarrow d \\
\tilde{X}_{\tilde{\Delta}}^{n} & \stackrel{\pi_{1}}{\rightarrow} & \tilde{X}
\end{array}
$$

We set $p=\operatorname{Id} \times d^{n-1}, q$ the projection onto the first factor, and $r=d \times$ $\mathrm{Id}^{n-1}$. Note that $\pi_{1}$ is flat and the square part of the diagram is a flat base change of $d$, so $\pi_{1}^{*} d_{*} \omega_{Y / \tilde{\Delta}}=r_{*} q^{*} \omega_{Y / \tilde{\Delta}}$. The projection formula tells us that $p_{*} p^{*}\left(q^{*} \omega_{Y / \tilde{\Delta}}\right)=p_{*} \mathcal{O}_{Y_{\tilde{\Delta}}^{n}} \otimes q^{*} \omega_{Y / \tilde{\Delta}}$. Since $p$ is a birational map of normal

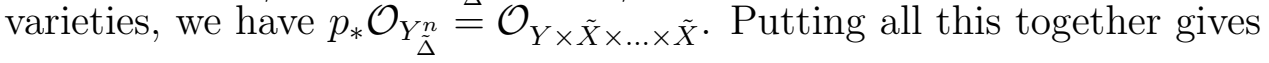

$$
\begin{aligned}
d_{*}^{n} \phi_{1}^{*} \omega_{Y / \tilde{\Delta}} & =(r \circ p)_{*}(q \circ p)^{*} \omega_{Y / \tilde{\Delta}} \\
& =r_{*} p_{*} p^{*} q^{*} \omega_{Y / \tilde{\Delta}} \\
& =r_{*}\left(p_{*} \mathcal{O}_{Y_{\tilde{\Delta}}^{n}} \otimes q^{*} \omega_{Y / \tilde{\Delta}}\right) \\
& =r_{*} q^{*} \omega_{Y / \tilde{\Delta}} \\
& =\pi_{1}^{*} d_{*} \omega_{Y / \tilde{\Delta}}
\end{aligned}
$$

This proves equation (2).

In the course of proving lemma 1 , recall that we established the equation:

$$
\omega_{\tilde{X}_{\Delta}^{n} / \tilde{\Delta}}^{[M]}=\pi_{1}^{*}\left(\omega_{\tilde{X} / \tilde{\Delta}}^{[M]}\right) \otimes \ldots \otimes \pi_{n}^{*}\left(\omega_{\tilde{X} / \tilde{\Delta}}^{[M]}\right)
$$


Using this along with (2) gives

$$
\begin{aligned}
\omega_{\tilde{X}_{\tilde{\Delta}}^{n} / \tilde{\Delta}}^{[M]} & =\pi_{1}^{*}\left(\omega_{\tilde{X} / \tilde{\Delta}}^{[M]}\right) \otimes \ldots \otimes \pi_{n}^{*}\left(\omega_{\tilde{X} / \tilde{\Delta}}^{[M]}\right) \\
& =\pi_{1}^{*}\left(d_{*} \omega_{Y / \tilde{\Delta}}^{M}\right) \otimes \ldots \otimes \pi_{n}^{*}\left(d_{*} \omega_{Y / \tilde{\Delta}}^{M}\right) \\
& =d_{*}^{n} \phi_{1}^{*} \omega_{Y / \tilde{\Delta}}^{M} \otimes \ldots \otimes d_{*}^{n} \phi_{n}^{*} \omega_{Y / \tilde{\Delta}}^{M} \\
& =d_{*}^{n} \omega_{Y_{\tilde{\Delta}}^{n} / \tilde{\Delta}}^{M}
\end{aligned}
$$

The last step is just the formula for the dualizing sheaf of a fiber product. This completes the proof of equation (1). We conclude that $\tilde{X}_{\tilde{\Delta}}^{n}$ has canonical singularities.

Before completing the proof, we need to fix some additional notation. Set

$$
G:=\operatorname{Gal}(\tilde{\Delta} / \Delta)
$$

and $j$ to be the map

$$
j: \tilde{X}_{\tilde{\Delta}}^{n} \longrightarrow X_{\Delta}^{n}
$$

induced by the base change. Let

$$
s: Z \longrightarrow \tilde{X}_{\tilde{\Delta}}^{n}
$$

be an equivariant desingularization of $\tilde{X}_{\tilde{\Delta}}^{n}$ with respect to the Galois action of $G$ (see [Hi]). Then we write the quotient map

$$
Q: Z \longrightarrow Z / G
$$

Note that $Z / G$ may be singular. Finally, the map from $Z$ to $X_{\Delta}^{n}$ is $G$ equivariant, so it factors through $Z / G$ giving a map

$$
R: Z / G \longrightarrow X_{\Delta}^{n}
$$

This is summarized in the following diagram:

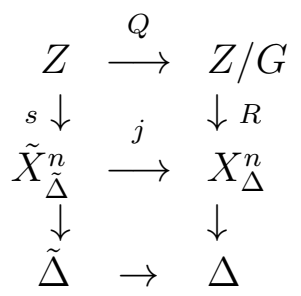


Now we show that $X_{\Delta}^{n}$ has canonical singularities. Let

$$
\alpha \in \Gamma\left(\omega_{X_{\Delta}^{n}}^{[m]}\right)
$$

be an $m$-pluricanonical form on $X_{\Delta}^{n}$. We want to show that $\alpha$ is a smooth form on $X_{\Delta}^{n}$, i.e. for any desingularization $V$ of $X_{\Delta}^{n}$, the pull back of $\alpha$ to $V$ is regular. It suffices to show that $R^{*} \alpha$ is a smooth form on $Z / G$, because a desingularization of $Z / G$ can also serve as a desingularization of $X_{\Delta}^{n}$. By an easy local computation, $j^{*} \alpha$ vanishes to order $m(|G|-1)$ along the central fiber of $\tilde{X}_{\tilde{\Delta}}^{n}$. Pulling back to the desingularization $Z$ (and increasing $m$ if necessary), we see that $s^{*} j^{*} \alpha$ is a smooth form vanishing to order $m(|G|-1)$ along the central fiber, because $\tilde{X}_{\tilde{\Delta}}^{n}$ has canonical singularities. The central fiber of $Z$ is precisely the fixed locus under the action of $G$. Therefore, we can apply the following lemma to $\theta=s^{*} j^{*} \alpha$ to show that it descends to a smooth form on $Z / G$ :

Lemma 2 Let $G$ be a finite group acting on the variety $Z$. Let $W$ denote a codimension d subvariety of $Z$ fixed pointwise by a subgroup $G_{W} \subset G$, and $\theta$ an invariant $m$-pluricanonical form. If $\theta$ vanishes to order at least $m\left(\left|G_{W}\right|-d\right)$ at every such $W$, then $\theta$ descends to a smooth form on $Z / G$,

For a proof of this, see CHM $\S 4.2$. We use $\beta$ to denote this smooth form on $Z / G$. Using the commutative diagram above, one can see that $\beta=R^{*} \alpha$, i.e. the pluricanonical form $\alpha$ pulls back to a smooth form on $Z / G$. This proves that $X_{\Delta}^{n}$ has canonical singularities.

To summarize, this proves the proposition in the special case of a one dimensional base. The proposition is a local statement on the base (in the analytic topology), so if it is true for families over a disc then it is true for general one dimension families. We use this as the base case for induction on the dimension of the base. We now prove the inductive step.

We will use the following result of Stevens( $[\mathbf{S t})$.

Theorem 5 Let $g: V \rightarrow \Delta$ be a family of proper varieties. Assume:

1. $V$ is a $\mathbb{Q}$-Gorenstein integral variety, and the fibers of $g$ are integral varieties.

2. The general fibers $g^{-1}(s)$ have only canonical singularities. 
3. The special fiber $g^{-1}(0)$ has log terminal singularities.

Then $V$ has canonical singularities.

We apply this theorem inductively to $V=X_{B}^{n}$ to reduce the dimension of the base. Note that $X_{B}^{n}$ has $\mathbb{Q}$-Gorenstein singularities by the lemma proven above. Choose a local analytic coordinate $y$ on $B$, and consider the level surfaces

$$
H_{s}=\{b \in B: y(b)=s\}
$$

for $s \in \Delta$. Assume that none of the $H_{s}$ are contained in the locus $S$ of surfaces with singularities worse than rational double points. Let

$$
h: X \rightarrow \Delta
$$

be the map associating $\left.X\right|_{H_{s}}=f^{-1}\left(H_{s}\right)$ to $s \in \Delta$. We also have the corresponding map

$$
g=h^{n}: X_{B}^{n} \rightarrow \Delta
$$

For all $s, H_{s} \cap S$ is again a Zariski closed proper subset of $H_{s}$, so the family

$$
f_{s}^{n}:\left.X\right|_{H_{s}} ^{n} \rightarrow H_{s}
$$

satisfies the hypotheses of Proposition 2. Applying the inductive hypothesis, we find that

$$
g^{-1}(s)=\left.X_{B}^{n}\right|_{H_{s}}=\left.X\right|_{H_{s}} ^{n}
$$

is canonical for all $s$. Since all the fibers of $g$ are canonical, and the total space $X_{B}^{n}$ is $\mathbb{Q}$-Gorenstein, we can apply Stevens' theorem to conclude that $X_{B}^{n}$ is also canonical. This concludes the proof that stable surface singularities are negligible.

\section{Proof of the Correlation Theorem}

In this section, we prove the correlation theorem for surfaces of general type (Theorem 1). We first prove a special case where the family has maximal variation of moduli and the singularities are not too bad. By definition, a family has maximal variation of moduli if there are no isotrivial connected subfamilies through the generic point. 
Theorem 6 (Correlation for Families with Maximal Variation) Let $X \rightarrow$ $B$ be a family of stable surfaces, with projective integral base and smooth general fiber. Assume that the associated map $\phi: B \rightarrow \overline{\mathcal{M}}$ is generically finite. Then there exists a positive integer $n$ such that $X_{B}^{n}$ is of general type.

Being of general type is a birational property, so there is no loss of generality if we take the base $B$ to be smooth. To show that $X_{B}^{n}$ is of general type for some large $n$, we must verify two statements:

1. $X_{B}^{n}$ has canonical singularities

2. $\omega_{X_{B}^{n}}$ is big

Note that the first statement is equivalent to saying that stable surface singularities are negligible, which was proved in the last section. The second statement allows us to get lots of pluricanonical differentials on $X_{B}^{n}$, and which pull back to a desingularization of $X_{B}^{n}$.

The key to the second statement is the following theorem:

Theorem 7 Let $f: X \rightarrow B$ be a family of surfaces, such that the general fiber is a surface of general type. Assume this family has maximal variation. Then for $m$ sufficiently large, we have that $f_{*} \omega_{X / B}^{m}$ is big.

This result is proven by Viehweg in [V2] (and more generally for arbitrary dimensional fibers by Kollár in [K2]). We need the following consequence of this result:

Proposition 4 Under the hypotheses of the theorem above, $\omega_{X_{B}^{n}}$ is big.

We will show that Theorem 7 implies Proposition 4 . Here $S^{[n]}$ will denote the reflexive hull of the $n$th symmetric power of a sheaf. To say that $f_{*} \omega_{X / B}^{m}$ is big means that for any ample line bundle $H$ on $B$ there exists an integer $n$ such that

$$
S^{[n]}\left(f_{*} \omega_{X / B}^{m}\right) \otimes H^{-1}
$$

is generically globally generated, i.e. the global sections of this sheaf generate over an open set of $B$. It is equivalent to say that this sheaf is generically globally generated for sufficiently large $n$. Now let $T^{[n]}$ denote the reflexive hull of the $n$th tensor power of a sheaf. We claim that for sufficiently large $n$

$$
T^{[n]}\left(f_{*} \omega_{X / B}^{m}\right) \otimes H^{-1}
$$


is generically globally generated. To prove this, we need a result from representation theory:

Proposition 5 Let $V$ be an $r$ dimensional vector space over a field of characteristic zero, and let $T^{n}(V)$ and $S^{q}(V)$ be the nth tensor power and qth symmetric power representations of $G l(V)$ respectively, and write $t=r !$. Then each irreducible component of $T^{n}(V)$ is a quotient of a representation

$$
S^{q_{1}}(V) \otimes \ldots \otimes S^{q_{t}}(V)
$$

where $q_{i} \geq \frac{n}{t+1}$.

This result is proved in [H2] for arbitrary 'positive' representations $T$ of $V$. This gives us a map

$$
\bigoplus S^{\left[q_{1}\right]}\left(f_{*} \omega_{X / B}^{m}\right) \otimes \ldots \otimes S^{\left[q_{t}\right]}\left(f_{*} \omega_{X / B}^{m}\right) \otimes H^{-1} \rightarrow T^{[n]}\left(f_{*} \omega_{X / B}^{m}\right) \otimes H^{-1}
$$

which is surjective over an open set of $B$. Let $H$ be ample and globally generated, and choose $n$ large enough to guarantee that each of the $S^{\left[q_{i}\right]}\left(f_{*} \omega_{X / B}^{m}\right) \otimes$ $H^{-1}$ is generically globally generated. This guarantees that the left hand side is generically globally generated, but then so is $T^{[n]}\left(f_{*} \omega_{X / B}^{m}\right) \otimes H^{-1}$.

Now we will prove that for some large $n$ the dualizing sheaf $\omega_{X_{B}^{n}}$ is big, i.e. for large $m$ we have

$$
h^{0}\left(X_{B}^{n}, \omega_{X_{B}^{n}}^{[m]}\right) \approx m^{(b+2 n)}
$$

where $b=\operatorname{dim}(B)$. We restrict ourselves to values of $m$ for which

1. $\omega_{X / B}^{[m]}$ is locally free.

2. $f_{*} \omega_{X / B}^{m}$ is big.

First we compute the canonical bundle of $X_{B}^{n}$ :

$$
\omega_{X_{B}^{n}}=\omega_{X_{B}^{n} / B} \otimes f^{n *} \omega_{B}=\pi_{1}^{*} \omega_{X / B} \otimes \ldots \otimes \pi_{n}^{*} \omega_{X / B} \otimes f^{n *} \omega_{B}
$$

As in lemma 1, taking $m$ th powers gives

$$
\omega_{X B}^{[m]}=\pi_{1}^{*} \omega_{X / B}^{[m]} \otimes \ldots \otimes \pi_{n}^{*} \omega_{X / B}^{[m]} \otimes f^{n *} \omega_{B}^{m}
$$


Applying $f_{*}^{n}$ to this gives

$$
\begin{aligned}
f_{*}^{n} \omega_{X}^{[m]} & =f_{*}^{n}\left(\pi_{1}^{*} \omega_{X / B}^{[m]} \otimes \ldots \otimes \pi_{n}^{*} \omega_{X / B}^{[m]}\right) \otimes \omega_{B}^{m} \\
& =f_{*}^{n} \pi_{1}^{*} \omega_{X / B}^{[m]} \otimes \ldots \otimes f_{*}^{n} \pi_{n}^{*} \omega_{X / B}^{[m]} \otimes \omega_{B}^{m} \\
& =T^{n}\left(f_{*} \omega_{X / B}^{[m]}\right) \otimes \omega_{B}^{m}
\end{aligned}
$$

Note this is also a reflexive sheaf. The inclusion map $\omega_{X / B}^{m} \rightarrow \omega_{X / B}^{[m]}$ induces a map of reflexive sheaves

$$
T^{[n]}\left(f_{*} \omega_{X / B}^{m}\right) \rightarrow T^{n}\left(f_{*} \omega_{X / B}^{[m]}\right)
$$

which is an isomorphism at the generic point of $B$.

Let $H$ be an invertible sheaf on $B$ so that $H \otimes \omega_{B}$ is very ample. By Viehweg's theorem and proposition 5 , we can choose $n$ so that $T^{[n]}\left(f_{*} \omega_{X / B}^{m}\right) \otimes$ $H^{-m}$ is generically globally generated for $m$ sufficiently large. The computations of the last paragraph show that $f_{*}^{n} \omega_{X_{B}^{n}}^{[m]} \otimes\left(H \otimes \omega_{B}\right)^{-m}$ is also generically globally generated for sufficiently large $m$. In particular, as this sheaf has rank on the order of $m^{2 n}$, there at least this many global sections. By our assumption on $H$, we have that $\left(H \otimes \omega_{B}\right)^{m}$ has on the order of $m^{b}$ sections varying horizontally along the base $B$. Tensoring, we get that $f_{*}^{n} \omega_{X_{B}^{n}}^{[m]}$ has on the order of $m^{2 n+b}$ global sections. Thus we conclude that

$$
h^{0}\left(\omega_{X_{B}^{n}}^{[m]}\right) \approx m^{2 n+b}
$$

This completes the proof of the proposition and the special case of the Correlation theorem.

Now we extend this special case to prove correlation for arbitrary families $f: X \rightarrow B$ of surfaces of general type. Since stable surface singularities are inclusive, after a generically finite base extension $B^{\prime} \rightarrow B$ every family of surfaces of general type dominates a family of stable surfaces $\psi: \mathcal{T}^{\prime} \rightarrow \Sigma^{\prime}$ with maximal variation:

$$
\begin{array}{ccccc}
X^{\prime} & \rightarrow & \mathcal{T}^{\prime} & \\
\downarrow & & \downarrow & \downarrow \\
B^{\prime} & \rightarrow & \Sigma^{\prime} &
\end{array}
$$

Take $n^{\text {th }}$ fiber products, where $n$ is chosen to ensure that $\mathcal{T}_{\Sigma^{\prime}}^{\prime n}$ is of general type. Use $X_{B^{\prime}}^{\prime}{ }^{n}$ to denote the component of the fiber product dominating 
$B^{\prime}$. We obtain a diagram:

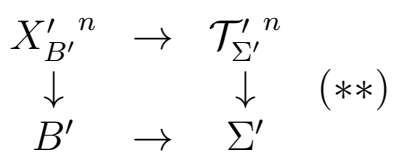

with $X_{B^{\prime}}^{\prime}{ }^{n}$ dominating $\mathcal{T}_{\Sigma^{\prime}}^{\prime}{ }^{n}$, a variety of general type. This shows that the correlation result holds if we allow ourselves to make a finite base change before we take the fiber products.

Now we show that the fiber products $X_{B}^{N} \rightarrow B$ dominate a variety of general type without taking a base change, provided $N$ is large enough. We will use the corollary at the end of $\S 3$ to construct our map. This corollary allows us to assume that the base extension $B^{\prime} \rightarrow B$ is Galois with Galois group $G$, and that $G$ acts birationally on the entire diagram $(*)$. That is, $G$ acts birationally on each of the varieties in $(*)$, and this action commutes with the morphisms of the diagram. It follows that $G$ acts naturally and birationally on $(* *)$, and taking quotients gives us

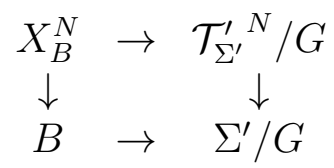

Setting $W=\mathcal{T}_{\Sigma^{\prime}}^{\prime} / G$, we obtain a rational dominant map

$$
h: X_{B}^{n} \longrightarrow W
$$

From the construction, we see that this map is generically finite when restricted to a general fiber of $X_{B}^{n} \rightarrow B$.

To conclude the proof, we need to show that $W=\mathcal{T}_{\Sigma^{\prime}}^{\prime}{ }^{N} / G$ is of general type, for some sufficiently large $N$. Specifically, we will show that enough of the $m$ pluricanonical differentials on $V=\mathcal{T}_{\Sigma^{\prime}}^{\prime N}$ descend to smooth differentials on $W=V / G$ to guarantee that $W$ is a variety of general type. First, note that $G$ acts faithfully on $\mathcal{T}^{\prime}$, but does not necessarily act faithfully on the base $\Sigma^{\prime}$. Let $G^{\prime}$ be the maximal quotient of $G$ acting faithfully on $\Sigma^{\prime}$, and set $g=|G|$. Let $\Phi_{1} \subsetneq \Sigma^{\prime}$ be the locus of points of $\Sigma^{\prime}$ with nontrivial stabilizer under the $G^{\prime}$ action, $\Phi_{2} \subsetneq \Sigma^{\prime}$ the locus on the base corresponding to fibers of $\mathcal{T}^{\prime} \rightarrow \Sigma^{\prime}$ fixed pointwise by a nontrivial subgroup of $G$. Let $D_{0}$ be an effective divisor on $\Sigma^{\prime}$ containing $\Phi_{1} \cup \Phi_{2}$, and $D$ the pullback of $g D_{0}$ to $V$. 
Note that for large $N$, the support of $D \subset V$ contains all the componenets of the fixed locus with codimension less than $g$. This is because the only components of the fixed point locus with small codimension correspond to fixed fibers of $\mathcal{T}^{\prime}$.

We repeat the proof we used in the maximum variation case, except that we choose positive $H$ so that $H \otimes \omega_{\Sigma^{\prime}}\left(-g D_{0}\right)$ is very ample. Again, we can choose $N$ so that

$$
\psi_{*}^{N} \omega_{V}^{[m]}(-m D)=T^{[N]}\left(\psi_{*}^{N} \omega_{\mathcal{T}^{\prime} / \Sigma^{\prime}}^{m}\right) \otimes \omega_{\Sigma^{\prime}}^{m}\left(-m g D_{0}\right)
$$

has $m^{(\operatorname{dim} V)}$ sections. This guarantees that

$$
h^{0}\left(\omega_{V}^{[m]}(-m D)\right) \approx m^{(\operatorname{dim} V)}
$$

In other words, there are lots of $m$ pluricanonical differentials on $V$ vanishing to high order along subvarieties of $V$ nontrivial stabilizer and codimension less than $g$. We apply lemma 2 of $\S 4$ to conclude that these forms descend to smooth forms on $W$, i.e. forms on $W$ that pull back to regular forms on a desingularization of $W$. Therefore $W$ is of general type and the Correlation Theorem is proved.

\section{Consequences of the Correlation Theorem}

We give some consequences of the Correlation Theorem. Many of these are stated in $\S 6$ of $\mathrm{CHM}$. The motivating conjectures can be found in $\mathbb{\square}$.

Recall the statement of the Geometric Lang Conjecture:

Conjecture 2 (Geometric Lang Conjecture) If $W$ is a variety of general type, the union of all irreducible, positive dimensional subvarieties of $W$ not of general type is a proper, closed subvariety $\Xi_{W} \subset W$.

We will call $\Xi_{W}$ the Langian exceptional locus of $W$. The following theorem describes how the Langian exceptional locus varies in families, if the geometric Lang conjecture is true.

Theorem 8 Assume the Geometric Lang Conjecture.

Let $f: X \rightarrow B$ be a flat family of surfaces in projective space, such that the general fiber is an integral surface of general type. Then there is a uniform 
bound on the degree of the Langian exceptional locus of fibers that are of general type i.e.

$$
\operatorname{deg}\left(\Xi_{X_{b}}\right) \leq D
$$

By Noetherian induction, it suffices to prove the bound on an open subvariety of $B$. Using the Correlation Theorem, for sufficiently high fiber products $f^{n}: X_{B}^{n} \rightarrow B$ we obtain a dominant rational map to a variety of general type

$$
\psi: X_{B}^{n} \rightarrow W
$$

We use $Y$ to denote the Langian exceptional locus of $W$, and $Z_{1}$ its preimage in $X_{B}^{n}$. Let $Z_{2}$ be the union of all positive dimensional fibers of the map

$$
X_{B}^{n} \rightarrow W \times B
$$

We set $Z=Z_{1} \cup Z_{2} ; Z$ is a proper subvariety of $X_{B}^{n}$.

Consider the projection map

$$
\pi_{n}: X_{B}^{n} \rightarrow X_{B}^{n-1}
$$

with fiber $\pi_{n}^{-1}(p)$ isomorphic to the stable surface $X_{f^{n-1}(p)}$. Since $Z$ is a proper subvariety of $X_{B}^{n}$, over an open set $U_{n-1} \subset X_{B}^{n-1}$ the fibers of $\pi_{n}$ are not contained in $Z$. For all $p \in U_{n-1}$, the degree of $Z$ restricted to the fiber $\pi_{n}^{-1}(p)$ is bounded. At the same time, the Langian locus $\Xi_{p}$ of any of these fibers is contained in $Z$, because for any component $C \subset \Xi_{p}$ either $\psi(C) \subset Y$ or $\psi(C)$ is a point. This concludes the proof.

This yields many remarkable corollaries. One example is the following

Corollary 2 Assume the Geometric Lang Conjecture. There exists a constant $D$ such that the sum of the degrees of all the rational and elliptic curves on a smooth quintic surface in $\mathbb{P}^{\nVdash}$ is less than D. In particular, there is a uniform bound on the number of rational and elliptic curves on a quintic surface.

Recently, Abramovich [AV] has found another proof of these results.

Now we shall discuss some number theoretic consequences of the Correlation Theorem. First, recall the Weak Lang Conjecture:

Conjecture 3 (Weak Lang Conjecture) If $W$ is a variety of general type defined over a number field $K$, then the $K$-rational points of $W$ are not Zariski dense in $W$. 
Assuming this conjecture, the Correlation Theorem implies the following:

Theorem 9 Assume the Weak Lang Conjecture.

Let $X \rightarrow B$ be a flat family of surfaces in projective space defined over a number field $K$ such that the general fiber is an integral surface of general type. For any $b \in B(K)$ for which $X_{b}$ is of general type, let $N(b)$ be the sum of the degrees of the components of $\overline{X_{b}(K)}$. Then $N(b)$ is uniformly bounded; in particular, the number of $K$ rational points not contained in the Langian locus is uniformly bounded.

The proof of this is similar to the proof of the previous theorem. Again we do induction on the dimension of the base $B$. First, we shall show that the rational points of the fibers must lie on a proper subscheme of bounded degree. Choose an integer $n$ so that there is a dominant rational map

$$
\psi: X_{B}^{n} \rightarrow W
$$

to a variety of general type $W$. Let $Y$ denote be a proper subvariety of $W$ that contains its $K$ rational points, and let $Z$ be its preimage in $X_{B}^{n}$. All the $K$ rational points of $X_{B}^{n}$ are contained in $Z$. We use

$$
\pi_{j}: X_{B}^{j} \rightarrow X_{B}^{j-1}
$$

to denote the projection morphisms. Finally, let $Z_{j}$ denote the maximal closed set in $X_{B}^{j}$ whose preimage in $X_{B}^{n}$ is $Z$, and let $U_{j}$ be the complement to $Z_{j}$. Note that $\pi_{j}^{-1}\left(Z_{j-1}\right) \subset Z_{j}$ by definition and that for $u \in U_{j-1}$ we have that $\pi_{j}^{-1}(u) \cap Z_{j}$ is a proper subvariety of $\pi_{j}^{-1}(u)$. We will use $d_{j}$ to denote the sum of the degrees of all the components of $Z_{j} \cap \pi_{j}^{-1}(u)$, regardless of their dimensions, and we set

$$
N=\max _{j}\left(d_{j}\right)
$$

If all the $K$ rational points of $B$ are concentrated along a closed subset, we are done by induction. Otherwise, pick a general $K$ rational point $b \in$ $B$. Let $j$ be the smallest integer for which $U_{j} \cap X_{b}^{j}(K)$ is empty, and let $u \in U_{j-1} \cap X_{b}^{j-1}(K)$. We have that $X_{b}=\pi_{j}^{-1}(u)$ and our set-up guarantees that $X_{b}(K) \subset Z_{j} \cap \pi_{j}^{-1}(u)$. In particular, since we have chosen everything generically, we find that $X_{b}(K)$ is contained in a subscheme of degree $N$.

Now we complete the proof. We have shown that the rational points on 
each fiber are concentrated along a subscheme of degree $N$. The components of this subscheme consist of points, rational and elliptic curves, and curves of higher genus. The rational and elliptic curves are contained in the Langian locus, so we ignore them, and there are at most $N$ components of dimension zero. Therefore, we just need the following lemma:

Lemma 3 Assume the Weak Lang Conjecture. Let $C$ be a (possibly singular) curve in projective space of degree $N$ defined over a number field $K$. Assume $C$ has no rational or elliptic components. Then there is a uniform bound on the number of $K$ rational points on $C$.

First, because the degree is bounded there are only finitely many possibilities for the geometric genera of the components of $C$. By the hypothesis, these genera are all at least two, so we can apply the uniform boundedness results for curves in CHM. This completes the proof of the theorem.

In the corollary that follows, quadratic points are points defined over some degree two extension of the base field.

Corollary 3 Assume the Weak Lang Conjecture. Fix a number field $K$, and an integer $g>2$. Then there is a uniform bound on the number of quadratic points lying on a non-hyperelliptic, non-bielliptic curve $C$ of genus $g$ defined over $K$.

Note that quadratic points on $C$ correspond to $K$ rational points on its symmetric square $\operatorname{Sym}^{2}(C)$. Moreover, a hyperelliptic (respectively bielliptic) system on $C$ corresponds to a rational (respectively elliptic) curve on $\operatorname{Sym}^{2}(C)(\mathrm{AH})$. In particular, the curves described in the theorem are pre-

cisely those for which $\Xi_{\mathrm{Sym}^{2}(C)}=\emptyset$, and so by the theorem $\# \operatorname{Sym}^{2}(C)(K)$ is finite and uniformly bounded.

\section{References}

[A] V. Alexeev. Boundedness and $K^{2}$ for log surfaces. Preprint.

[AH] D. Abramovich, J. Harris. Abelian varieties and curves in $W_{d}(C)$. Compositio Math. 78 (1991) 227-238.

[AV] D. Abramovich, J.F. Voloch. Lang's conjectures, fibered powers, and uniformity. Preprint. 
[CHM] L. Caporaso, J. Harris, B. Mazur. Uniformity of rational points. Preprint to appear in J. Amer. Math. Soc.

[H] R. Hartshorne. Stable reflexive sheaves. Math. Ann. 254 (1980) 121-176.

[H2] R. Hartshorne. Ample vector bundles. Publ. Math. IHES 29 (1966) 6394.

[Hi] H. Hironaka. Idealistic exponents of singularity. In: Algebraic Geometry, J.J. Sylvester Symposium, Johns Hopkins Univ., Baltimore, Md., 1976, Johns Hopkins Univ. Press, Baltimore 1977, 52-125.

[I] S. Iitaka. Birational Geometry for Open Varieties. Sém. Math. Sup. Montreal 76, Presses de l'Universite de Montreal, Montreal 1981.

[Ka] Y. Kawamata. Semistable minimal models of threefolds in Positive or Mixed Characteristic. J. Algebraic Geometry 3 (1994) 463-491.

[KKMS] G. Kempf, F. Knudsen, D. Mumford, B. Saint Donat. Toroidal Embeddings. Lect. Notes Math. 339 (1973).

[K] J. Kollár. Projectivity of complete moduli. J. Differential Geometry 32 (1990) 235-268.

[K2] J. Kollár. Subadditivity of the kodaira dimension: fibers of general type. In: Algebraic Geometry, Sendai, 1985, T. Oda ed., Adv. Stud. in Pure Math. 10, North Holland, New York 1987, 361-398.

[K-SB] J. Kollár, N. Shepherd-Barron. Threefolds and deformations of surface singularities. Invent. math. 99 (1988) 299-338.

[L] S. Lang. Hyperbolic and diophantine analysis. Bull. Amer. Math. Soc. 14, No. 2 (1986) 159-205.

[R] M. Reid. A young person's guide to canonical singularities. Proceedings of Symposia in Pure Mathematics 46 (1987) 345-414.

[St] J. Stevens. On canonical singularities as total spaces of deformations. Abh. Math. Sem. Univ. Hamburg 58 (1988) 275-283. 
[V] E. Viehweg. Weak positivity and the additivity of the Kodaira dimension for certain fibre spaces. In: Algebraic varieties and analytic varieties, S. Iitaka ed., Adv. Stud. in Pure Math. 1, North Holland, New York 1983, 329-353.

[V2] E. Viehweg Weak positivity and the additivity of the Kodaira dimension, II:The local Torelli map. In: Classification of Algebraic and Analytic Manifolds, Kenji Ueno ed., Progress in Mathematics 39, Birkhäuser, Boston 1983, 567-589.

Brendan Hassett

Department of Mathematics, Harvard University

1 Oxford Street, Cambridge, MA 02138

hassett@math.harvard.edu 